\title{
Rechtsgeschichte
}

http://www.rg-rechtsgeschichte.de/rg3

$\operatorname{Rg} 32003 \quad 66-67$

Zitiervorschlag: Rechtsgeschichte Rg 3 (2003)

http://dx.doi.org/10.12946/rg03/066-067

\section{Reinhard Zimmermann}

Gegenwartsbedeutung 


\section{Gegenwartsbedeutung}

\author{
28. Februar 2003
}

Sehr geehrter Herr Kiesow,

haben Sie vielen Dank für Ihren Brief vom 20. Februar.

Sie fragen »Wozu Rechtsgeschichte? « Das ist in der Tat eine spannende Frage und zudem eine solche, die sich wohl jeder Rechtshistoriker selbst aus vielen Anlässen und bei vielen Gelegenheiten vorgelegt hat. Eine allgemein gültige Antwort gibt es darauf nicht. Überspitzt ließe sich sagen, dass es auf die Frage so viele Antworten gibt, wie es Rechtshistoriker gibt. Hinzukommt, dass auch ein einzelner Rechtshistoriker mit verschiedenen Arbeiten durchaus verschiedene Zwecke verfolgen kann. Die Erkenntnisinteressen können variieren, und dasselbe gilt für die mit rechtshistorischer Forschung verfolgten Zwecke.

Mir persönlich ist es immer legitim erschienen, die Frage nach dem Sinn und Zweck von Rechtsgeschichte zu stellen. Ich denke sogar, dass rechtshistorische Forschung in der Regel aus einem bestimmten Erkenntnisinteresse heraus, und das heißt doch im Grunde auch: zu einem bestimmten "Zweck « betrieben wird. Für ebenso legitim habe ich andererseits aber auch immer die Bemühungen um ein möglichst zweckfreies Gewinnen von Erkenntnissen über die Vergangenheit gehalten. Ich hebe dies eigentlich nur deshalb hervor, weil die Redaktion Ihrer Vorgängerzeitschrift gegenüber der Frage »Wozu Rechtsgeschichte? « merkwürdig intolerant war. Insofern freut es mich, dass auch Sie diese Frage nunmehr für nützlich, legitim, ja sogar spannend halten.
Einer Reihe meiner Arbeiten liegt die Auffassung zugrunde, dass rechtshistorische Erkenntnisse das Verständnis des geltenden Rechts erleichtern können, ja mitunter geradezu erst ermöglichen: Rechtsgeschichte kann insofern eine Gegenwartsbedeutung haben. Sie kann uns zeigen, wie und warum sich das Recht so entwickelt hat, wie wir es heute vorfinden. Daraus lassen sich unter anderem Anstöße und Argumente (wenngleich natürlich keine normativen Vorgaben) für die Interpretation und Fortbildung des modernen Rechts gewinnen. Diese Aufgabe der Rechtsgeschichte ist besonders wichtig in Situationen des Umbruchs. Einen derartigen Umbruch erleben wir momentan in den mir besonders nahe liegenden Bereichen des Privatrechts und der Privatrechtswissenschaft: Beide Bereiche werden umfassend »europäisiert«. In diesem Zusammenhang scheint mir die Frage wichtig und sinnvoll, wie »europäisch « unser Privatrecht eigentlich bereits ist: inwieweit, mit anderen Worten, die modernen nationalen Rechtsordnungen in Europa auf gemeinsamen historischen Grundlagen beruhen. Damit verbunden ist gleichzeitig die Frage, inwieweit und warum diese Rechtsordnungen sich in vielen Punkten aber auch unterschiedlich entwickelt haben. Die rechtshistorische hat hier vor allem auch eine rechtsvergleichende Dimension, und nicht zuletzt deshalb sind mir Rechtsgeschichte und Rechtsvergleichung immer, wie Herr Kötz dies einmal formuliert hat, als Zwillingsschwestern erschienen. Jedenfalls gehören diese Disziplinen für mich unmittelbar zusammen, da sich erst so das spezifische Profil der in den modernen Gesetzbüchern gewählten (oder auch im common law bevorzugten) Lösungswege ergibt. 
Diese hier nur in gröbsten Umrissen skizzierten Gedanken habe ich bereits mehrfach versucht darzulegen und, was mir wichtiger erscheint, in der praktischen, rechtshistorischrechtsvergleichenden Arbeit zu erproben (vgl. zuletzt etwa noch einmal AcP 202 [2002] 243 ff.). Einzelne dieser Arbeiten haben durchaus auch die Aufmerksamkeit Ihrer Zeitschrift und zuvor des Rechtshistorischen Journals gefunden. Auf dem Monte Verità bei Ascona hat übrigens vor einigen Jahren sogar bereits ein (von den Herren Kollegen Pio Caroni und Gerhard Dilcher dokumentiertes) Symposium stattgefunden, auf dem die hier skizzierte Antwort auf die Frage »Wozu Rechtsgeschichte? « eingehend diskutiert worden ist. Ferner ist die Frage eines möglichen Nutzens der Rechtsgeschichte für das moderne Recht in dem von mir mit- herausgegebenen und Hans Hermann Seiler gewidmeten Band »Rechtsgeschichte und Privatrechtsdogmatik « thematisiert worden. Um den »Praktischen Nutzen der Rechtsgeschichte « geht es in einem soeben erschienenen Band zu Ehren von Hans Hattenhauer; meine Position habe ich dort auf S. 6oI ff. noch einmal beschrieben.

Ich könnte meine Sicht der Dinge im Rahmen Ihrer Essaysammlung nur noch weiteres Mal wiederholen. Dies erscheint mir nicht sinnvoll, und ich bitte Sie deshalb sehr herzlich um Verständnis dafür, wenn ich Ihnen kein Manuskript einreiche.

Mit freundlichen Grüßen und mit den besten Wünschen bin ich

Ihr

Reinhard Zimmermann
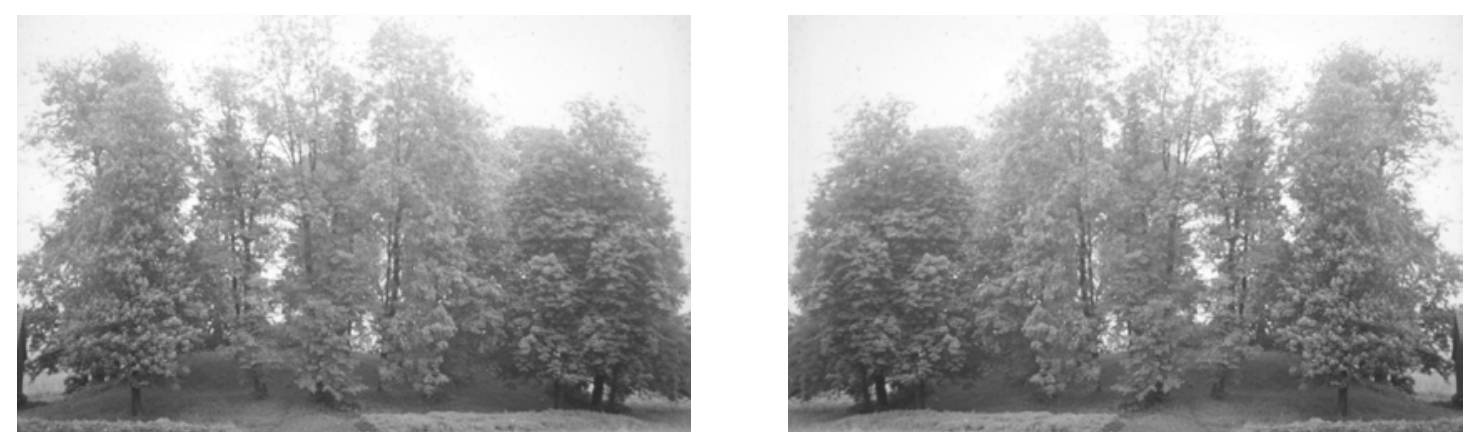